\title{
Identification of global regulators of T-helper cell lineage specification
}

\author{
Kartiek Kanduri ${ }^{1,2}$, Subhash Tripathi ${ }^{1}$, Antti Larjo ${ }^{2}$, Henrik Mannerström² ${ }^{2}$, Ubaid Ullah', Riikka Lund ${ }^{1}$, \\ R. David Hawkins ${ }^{1,3,4}$, Bing Ren ${ }^{5,6}$, Harri Lähdesmäki ${ }^{2^{*+}}$ and Riitta Lahesmaa ${ }^{1 *+}$
}

\begin{abstract}
Background: Activation and differentiation of T-helper (Th) cells into Th1 and Th2 types is a complex process orchestrated by distinct gene activation programs engaging a number of genes. This process is crucial for a robust immune response and an imbalance might lead to disease states such as autoimmune diseases or allergy. Therefore, identification of genes involved in this process is paramount to further understand the pathogenesis of, and design interventions for, immune-mediated diseases.

Methods: We aimed at identifying protein-coding genes and long non-coding RNAs (IncRNAs) involved in early differentiation of T-helper cells by transcriptome analysis of cord blood-derived naïve precursor, primary and polarized cells.

Results: Here, we identified lineage-specific genes involved in early differentiation of Th1 and Th2 subsets by integrating transcriptional profiling data from multiple platforms. We have obtained a high confidence list of genes as well as a list of novel genes by employing more than one profiling platform. We show that the density of lineage-specific epigenetic marks is higher around lineage-specific genes than anywhere else in the genome. Based on next-generation sequencing data we identified lineage-specific IncRNAs involved in early Th1 and Th2 differentiation and predicted their expected functions through Gene Ontology analysis. We show that there is a positive trend in the expression of the closest lineage-specific IncRNA and gene pairs. We also found out that there is an enrichment of disease SNPs around a number of IncRNAs identified, suggesting that these IncRNAs might play a role in the etiology of autoimmune diseases.
\end{abstract}

Conclusion: The results presented here show the involvement of several new actors in the early differentiation of T-helper cells and will be a valuable resource for better understanding of autoimmune processes.

\section{Background}

CD4+ T-helper (Th) cells are critical players in adaptive immune responses and protect the host against various pathogens. Naive CD4+ $\mathrm{T}$ cells are multi-potent in nature and have an ability to differentiate into distinct effector and regulatory subtypes that express lineagespecific regulators, including transcription factors and signature cytokines. For example, Th1 cells express the master transcription factor gene $T B X 21$ and secrete interferon $\gamma$ and Th2 cells express GATA3 and secrete

\footnotetext{
* Correspondence: harri.lahdesmaki@aalto.fi; riitta.lahesmaa@btk.fi

${ }^{\dagger}$ Equal contributors

${ }^{2}$ Department of Computer Science, Aalto University School of Science,

Espoo, Finland

'Turku Centre for Biotechnology, University of Turku and Åbo Akademi

University, Turku, Finland

Full list of author information is available at the end of the article
}

interleukin (IL)4 and IL13 cytokines. Because these effector T-helper cell lineages are crucial for mounting distinct immune responses, inappropriate execution of their differentiation processes may result in imbalance between T-helper cell subsets and ultimately lead to various inflammatory autoimmune diseases and allergic responses [1-3]. To understand and develop potential therapeutic treatment regimes, it is important to get a high-resolution map of regulators involved in T-helper cell differentiation. Previous studies have identified elements involved in T-helper cell differentiation [4-8].

Lineage-specificity is a dynamic process that involves molecular mechanisms resulting in the expression of genes that establish lineage-specific gene expression and/or suppress alternative developmental fates. Transcriptional regulation is one way of achieving lineage- 
specificity. Only a small portion of RNA is translated into proteins, although vast chunks of human DNA are transcribed $[9,10]$. These translated mRNAs are designated protein-coding genes. Epigenetic mechanisms represent the second layer of lineage-specific gene expression and involve histone modification, DNA methylation and non-coding RNAs [11-14]. We have previously shown that lineage-specific enhancer elements are at work in driving the expression of lineage-specific genes in Th1 and Th2 cells [15]. Long non-coding RNAs (lncRNA) are non-coding RNAs that are more than 200 nucleotides in length and do not have an open reading frame [16]. Recent studies show that non-coding RNAs that are not translated appear to be part of the vast regulatory machinery $[17,18]$.

In this study, we aimed at identifying lineage-specific mRNAs and lncRNAs participating in early differentiation (72 h) of Th1 and Th2 cells by comparing them to naïve (Thp) and activated CD4+ T cells (Th0). We utilized transcriptional profiling data from three different profiling platforms to get a high-confidence list of genes involved in T-helper cell lineage specification. By employing next-generation sequencing techniques, we were able to identify genes that were not previously known in the context of T-helper cell differentiation. Utilizing the same sequencing data, we were able to determine lineage-specific lncRNAs involved in early Thelper cell differentiation. We observed that there is a positive trend in the expression of lineage-specific lncRNAs lying in the vicinity of lineage-specific genes. In addition, using genome-wide data on histone modifications from Th1 and Th2 cells at $72 \mathrm{~h}$, we also found that lineage-specific enhancers and promoters are more preferentially located around lineage-specific genes/ lncRNAs than anywhere else in the genome. This shows the highly selective nature of the regulatory elements involved in T-helper cell differentiation. In addition, we further characterized lineage-specific IncRNAs for their predicted functions through Gene Ontology (GO) analysis using an lncRNA-mRNA co-expression network. This will be a valuable resource for further studies since the function of majority of lncRNAs is unknown.

\section{Methods}

\section{Ethics statement}

This study was approved by the Ethics Committee of the Hospital district of Southwest Finland in line with the 1975 Declaration of Helsinki. Informed consent was obtained from each donor.

\section{Human cord blood CD4+ T-cell isolation and culturing}

Naive CD4+ T cells were isolated from human umbilical cord blood of healthy neonates born in Turku University Central Hospital. Mononuclear cells were isolated using
Ficoll-Paque gradient centrifugation (Amersham Pharmacia Biotech, Uppsala, Sweden) and CD4+ T cells were purified using positive selection (Dynal CD4 Positive Isolation Kit, Invitrogen, Carlsbad, CA, USA). CD4+ T cells from several individuals were pooled after the isolation. Purified CD4+ T cells were cultured in Yssel's medium (Iscove's modified Dulbecco's medium supplemented with Yssel medium concentrate plus penicillin/streptomycin) supplemented with $1 \%$ human $\mathrm{AB}$ serum (Red Cross Finland Blood Service). Cells were activated with platebound anti-CD3 $(2.5 \mu \mathrm{g} / \mathrm{ml})$ and soluble anti-CD28 $(500 \mathrm{ng} / \mathrm{ml}$; both were from Immunotech, Marseille, France). Simultaneously, Th1 polarization was initiated with $2.5 \mathrm{ng} / \mathrm{ml}$ IL12 and Th2 neutralizing antibody antiIL4 $(1 \mu \mathrm{g} / \mathrm{ml})$; Th2 differentiation was promoted using $10 \mathrm{ng} / \mathrm{ml}$ IL4 plus Th1 neutralizing antibody antiinterferon $\gamma(1 \mu \mathrm{g} / \mathrm{ml})$ (all antibodies from R\&D Systems, Minneapolis, MN, USA); or cells were cultured with only neutralizing antibodies (anti-interferon $\gamma$ and anti-IL4) and without polarizing cytokines (Th0 cells). IL2 $(40 \mathrm{U} / \mathrm{ml}$, $R \& D$ Systems) was added on the second day of culture. Further, cells were supplemented with media and divided every second day to keep the polarizing conditions during the culture until day 7 . The polarization was verified by checking the expression of polarization marker genes for Th1 and Th2 subsets.

\section{RNA isolation and transcriptional profiling}

Total RNA was extracted from naïve precursor human cord blood CD4+ T cells, activated Th0 cells, and differentiated Th1 and Th2 cells at $72 \mathrm{~h}$ using Trizol reagent (Invitrogen). For hybridization on the Affymetrix Human Genome U133 Plus 2.0 array, 250 ng of total RNA was used as starting material and was processed with an Affymetrix GeneChip 3' IVT Express kit according to the sample preparation guide. For hybridization on the Illumina HumanHT-12 v4 Expression BeadChip, 300 ng of total RNA was used as starting material and was processed with an Illumina TotalPrep RNA Amplification kit according to the sample preparation guide. For sequencing, $400 \mathrm{ng}$ of total RNA was used as starting material and libraries were prepared with an Illumina TrueSeq RNA Sample Prep kit v2 according to the sample preparation guide. The sequencing data were generated using an Illumina HiSeq-2000 instrument and the number of reads obtained can be found in Additional file 1. These transcriptional profiling data have been deposited in Gene Expression Omnibus (GEO) under accession [GEO:GSE71646].

\section{Analysis of Affymetrix microarray data}

The $R$ statistical environment was used for analysis. Affymetrix microarray data were normalized using the robust multi-array average algorithm implemented in 
the affy package [17]. Duplicate and un-annotated probes were removed using the genefilter package [19]. The probeset with the highest inter-quartile range was retained in case of duplicates. Present and absent calls for probesets were generated by fitting the chip-wide log2-transformed expression data to a two-component Gaussian distribution function, using the standard Expectation-Maximization (EM) algorithm implemented in the mixtools package [20]. A probeset was defined to be present if the corresponding data point had a higher likelihood for the Gaussian component with a higher mean value in all the replicates of the sample subtype [21]. Differential expression analysis was done using moderated unpaired t-test as implemented in limma [22]. The genes were considered as differentially expressed if the Benjamini-Hochberg adjusted $p$ value $<0.05$ and $\log 2$ fold-change $<-1$ or $>1$.

\section{Analysis of Illumina microarray data}

The R statistical environment was used for analysis. Illumina microarray data were preprocessed, including background adjustment, variance stabilization transformation and quantile normalization as implemented in the lumi package [23]. Duplicate and un-annotated probes were removed using the genefilter package [19]. The probeset with the highest inter-quartile range was retained in case of duplicate probesets. Present and absent calls were obtained using the detection $p$ value. A probeset was defined to be present if the detection $p$ value $<0.01$ in all the replicates of a sample subtype. Differential expression analysis was performed as described in analysis of Affymetrix microarray data.

\section{Analysis of RNA-sequencing data for gene expression}

The quality of sequenced reads was checked using FastQC [24] and the reads were mapped to the hg19 reference transcriptome and genome build using TopHat [25]. Gene counts were obtained using the htseq-count script included in the htseq tool. Raw counts were normalized and variance-stabilized values were obtained using methods implemented in the DESeq package [26] in R. Present and absent calls were generated by fitting the normalized values to a two-component Gaussian distribution function using the EM algorithm implemented in the mixtools package in R [20]. A gene was defined to be present if the corresponding data point had a higher likelihood for the Gaussian component with a higher mean value in all the replicates of the sample subtype. Differential expression analysis was done on raw counts using the default settings in the DESeq package. The genes were considered to be differentially expressed if the Benjamini-Hochberg adjusted $p$ value $<0.05$ and modified $\log 2$ fold-change $<-1$ or $>1$. The resulting genes were refined using the previously generated present and absent calls.

\section{Analysis of RNA-sequencing data to identify IncRNAs}

Using the reads mapped to the hg19 reference genome, we estimated the expression levels of lncRNAs using the htseq-count script included in the htseq tool by providing the genomic features from the GENCODE v16 catalog of lncRNAs [27] along with the transcriptome. Differential expression of lncRNAs was done on raw counts using the default settings in the DESeq package [26]. The lncRNAs were considered to be differentially expressed if the Benjamini-Hochberg adjusted $p$ value $<0.05$ and modified $\log 2$ fold-change $<-1$ or $>1$. We define a lineage-specific lncRNA to be in the vicinity of a lineage-specific gene if it is within $5 \mathrm{~kb}$ upstream or $30 \mathrm{~kb}$ downstream of the gene.

\section{Lineage-specific genes or IncRNAs}

We selected all the genes that are differentially expressed in Thp versus Th0, Th1 and Th2 subsets from the three platforms and made a confident list of differentially expressed genes by checking that each gene was differentially expressed in at least two or more platforms with the same directionality in their fold change. In cases of novel genes or lncRNAs, we used the above comparisons from next-generation sequencing data only. We defined a feature to be Th1- or Th2-specific if it is uniquely differentially expressed in only Thp versus Th1 or Thp versus Th2 comparisons, respectively, but not differentially expressed in Thp versus Th0.

\section{Th1- and Th2-specific enhancer and promoter marks around lineage-specific genes/IncRNAs}

We overlaid enhancer marks found in Th1 and Th2 cells from a previously published study [15] on lineage-specific genes/lncRNAs obtained in this study. We define an enhancer mark to be in the vicinity of a lineage-specific feature if it is within $125 \mathrm{~kb}$ upstream or downstream of the transcription start site of the feature. We also overlaid promoter marks found in Th1 and Th2 cells obtained from the same dataset on lineage-specific genes/lncRNAs. We define a promoter mark to be in the vicinity of a lineage-specific feature if it is within $2.5 \mathrm{~kb}$ upstream or downstream of the transcription start site of the feature. For randomization tests, we randomly $(n=10,000)$ picked the same number of genes as that of a lineage-specific set from anywhere else in the genome and quantified the overlap of enhancer and promoter marks around them. The $p$ values were computed with respect to this randomly generated null distribution.

\section{Prediction of GO terms for IncRNAs}

In order to predict GO terms for IncRNAs, we constructed a co-expression network of lncRNAs and protein-coding 
genes. We defined a lncRNA to be co-expressed with a protein-coding gene if the absolute Pearson's correlation coefficient between their expression is greater than 0.9. For each group of protein-coding genes which are coexpressed with a particular lncRNA gene, we performed a topology based GO enrichment test as implemented in the topGO package in R [28]. Specifically, we used Fisher's exact test and then attributed the enriched GO terms with a $p$ value of $<0.01$ to that specific lncRNA.

\section{Disease-associated single nucleotide polymorphism analysis}

Disease-associated single nucleotide polymorphism (SNP) data were obtained from the National Center for Biotechnology Information (http://www.ncbi.nlm.nih.gov/projects/ gapplusprev/sgap_plus.htm). All SNPs with a $p$ value $>1 \mathrm{e}$ 5 were excluded from further analysis. A gene was defined to be associated with a SNP if it is within $\pm 100 \mathrm{~kb}$ of the SNP. Enrichment analysis of traits was performed using hypergeometric distribution.

\section{Results}

Transcriptional analysis of Th1- and Th2-specific genes

Cellular differentiation to a specific subset requires activation of cell type-specific genes and suppression of genes of alternative lineages. To identify the lineage-specific genes, we analyzed transcriptional data for differential gene expression for Thp versus Th0, Th1 and Th2 subsets (Additional file 2). The number of genes determined to be present and available for analysis was 11,753 for Affymetrix arrays, 9210 for Illumina arrays and 13,744 for Illumina Sequencing (Figure S2a in Additional file 3). The transcriptomic platform comparison results are provided in Figure S2b, c in Additional file 3, and in Additional files 4,5 , and 6. According to our definition of lineage specificity and based on the data from the three platforms, there are 249 Th1-specifying genes and 491 Th2-specifying genes (Fig. 1; Additional file 7). These are confident lists of lineage-specific genes and have been internally validated as they are obtained from multiple sources. We also obtained a novel list of lineage-specific genes using next-generation sequencing data, in which there are 189 Th1-specific genes and 272 Th2-specific genes (Additional file 8). Among the lineage-specific genes, our analysis identified those encoding cytokines, chemokines, chemokine receptors, enzymes and transcription factors. Additionally, we found a panel of genes that were upregulated and down-regulated in a lineage-specific manner. The Th1-specific ones include genes with both known and novel roles in Th1 cell differentiation. For example, GIMAP4, CCL3, CXCR5, FUT7, IL21, TBKBP1, ABHD5 and $A P O B E C 3 G$ were up-regulated and $B A C H 2, C S T L$, $A F F 3, T G F B 3$ and MAL were down-regulated specifically in the Th1 cell lineage. FUT7, an enzyme that catalyzes

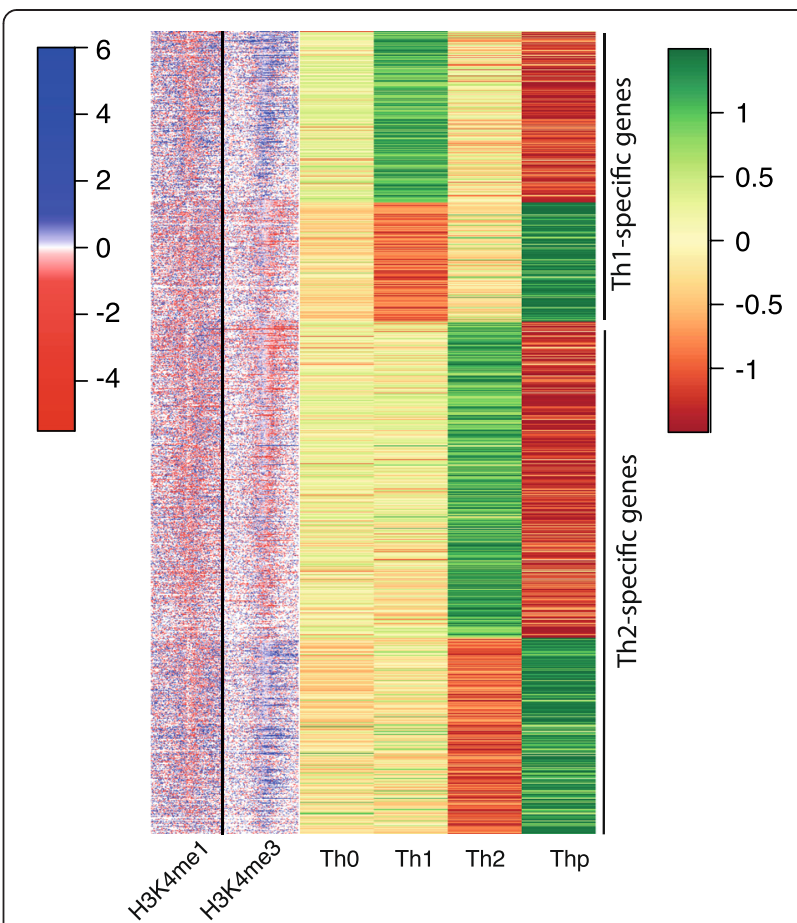

Fig. 1 Th1- and Th2- specific genes and their associated epigenetic marks in the human genome. Heatmap showing gene expression and epigenetic profiles of Th1- and Th2-specific genes in T-helper subsets. The figure shows both high confidence and novel genes. Rows were first ordered based on log2 fold change and then by expression value. Normalized expression from sequencing data was standardized using Z-score for visualization purposes. In the case of H3K4me 1 and H3K4me3 marks, the ratio of tag counts at the transcription start site between Th1 and Th2 is shown

synthesis of sialyl Lewis ${ }^{\mathrm{X}}$ antigens, has been shown to be expressed in CD4+ T cells [29]. Additionally it has binding sites for both GATA-3 and T-bet, master transcription factors for Th1 and Th2 cells where T-bet induces and GATA-3 inhibits the transcription of the FUT7 gene [30]. CCL3 (MIP-1 $\alpha)$ has previously been shown to be associated with the type 1 immune response [31]. CXCR5 is a chemokine receptor expressed on follicular T-helper cells. $A P O B E C 3 G$ expression is regulated in different CD4+ T-helper cells and is critical for modulation of HIV infectivity [32, 33]. TBKBP1 is involved in TNF$\alpha-N F-k B$ interaction and potentially has a critical role in antiviral innate immunity [34]. Genes down-regulated in response to Th1 differentiation and whose expression is increased in alternative lineages include CSTL, AFF3, and TGFB3, which are expressed in Th17 cells [35], and $B A C H 2$ and $M A L$, which are expressed in Th2 cells [36]. Th2 marker genes include those encoding the transcription factors GATA3 and GFI1 and lineage-specific cytokines, e.g., IL13, CCL17, and CCL20 [37-40]. Other genes include THY1, NOD2, SOCS1, ABHD6, PPP1R14A, PPARG, and BCAR3. The role of THY1 and NOD2 has 


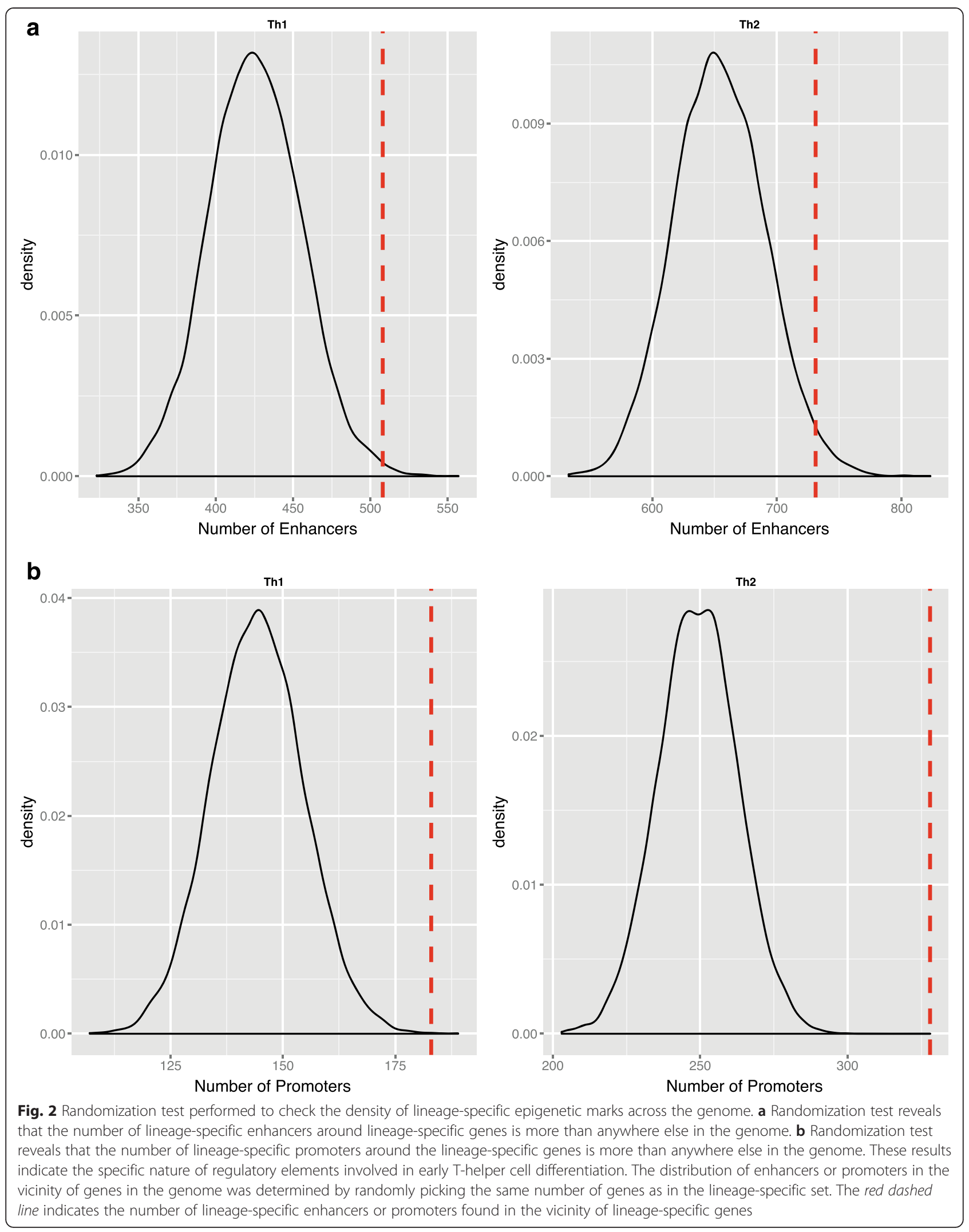


been documented in Th2 differentiation [41-43]. However, the role of ABHD6, PPP1R14A, PPARG, and BCAR3 in Th2 development remains to be determined.

We further validated the lineage specificity of these genes using lineage-specific enhancers and promoters. Enhancers and promoters were previously found to be differentially methylated at lysine 4 of histone $\mathrm{H} 3$ proteins [44]. We expected to find more active enhancer and promoter marks around lineage-specific genes than anywhere else in the genome. In order to determine the lineagespecific enhancers around lineage-specific genes, we overlaid lineage-specific enhancers from a previous study [15]. We found 508 Th1 enhancers around Th1-specific genes and 731 Th2 enhancers around Th2-specific genes (Fig. 2a). We then performed a randomization experiment $(10,000$ times) to compare the density of lineage-specific enhancers with that anywhere else in the genome. We found that there are more lineage-specific enhancers around lineage-specific genes than anywhere else in the genome $($ Th1 $p$ value $=0.0038$; Th2 $p$ value $=0.0196$; Fig. 2a). We repeated the same procedure with active promoters and found out that there are 183 Th1 active promoters, defined by the presence of both H3K4me3 and H3K27ac marks, around Th1-specific genes and 328 Th2 active promoters around Th2specific genes. Randomization test results showed that there are more lineage-specific active promoters around lineage-specific genes than anywhere else in the genome (Th1 $p$ value $=0.0003$; Th2 $p$ value $<10^{-4}$ ). These findings suggest the specific nature of genes and their epigenetic marks in T-helper cell differentiation.

We also looked for overlap between disease-associated SNPs and lineage-specific genes found in this study to explore their role in immune-mediated diseases. SNPs belonging to immune-mediated diseases, including asthma and Hodgkin disease, were found to be enriched in Th2-specific genes. Additionally, we found that SNPs belonging to other diseases were also enriched in Th1and Th2-specific genes (Table 1).

\section{Identification of lineage-specific IncRNAs in Th1 and Th2 subsets}

In order to find lineage-specific IncRNAs, we determined differentially expressed lncRNAs between Thp versus Th0, Th1 and Th2 subsets. By our definition of lineage specificity, there are 136 Th1 lineage-specific lncRNAs and 181 Th2 lineage-specific lncRNAs (Fig. 3a; Additional file 9). These lineage-specific lncRNAs can be classified into antisense (152), intergenic (83), processed transcript (62), sense intronic (15), sense overlapping (4) and 3' overlapping (1) based on their location in the genome. In accordance with previous studies [45], we observed that lncRNAs have lower expression than protein coding genes (Additional file 10). However, lineage-
Table 1 Enrichment of disease-associated SNPs in Th1- and Th2-specific genes

\begin{tabular}{lc}
\hline Disease & $P$ value \\
\hline Th1-specific genes & \\
Endometriosis & 0.0016 \\
Ovarian neoplasms & 0.0087 \\
Narcolepsy & 0.0311 \\
Th2-specific genes & \\
Hodgkin disease & 0.0119 \\
Moyamoya disease & 0.0256 \\
Osteoarthritis & 0.0256 \\
Asthma & 0.0259 \\
Osteoarthritis, knee & 0.0393 \\
Diabetes mellitus, type 2 & 0.0481 \\
\hline
\end{tabular}

specific lncRNAs are expressed at a higher level than the rest of the lncRNAs (Additional file 10) as reported in a recent study [46]. We then looked for lineage-specific lncRNAs that are in the vicinity of lineage-specific genes. There are 24 Th1 lineage-specific lncRNAs around Th1 lineage-specific genes and 47 Th2 lineage-specific lncRNAs around Th2-specific genes (Additional file 11). We observed a positive trend between the expression of these lineage-specific lncRNAs and lineage-specific genes (Fig. 3b).

We also looked at the relationship between lineagespecific lncRNAs and epigenetic marks that lie in their vicinity. We followed the same approach as that used for lineage-specific genes to determine enhancers and the epigenetic state of promoters around lineage-specific lncRNAs; 392 Th1 enhancers and 53 Th1 promoters were found in the vicinity of Th1-specific lncRNAs and 372 Th2 enhancers and 61 Th2 promoters were found in the vicinity of Th2-specific lncRNAs. Interestingly, the H3K4me1 and H3K4me3 histone mark maps in Fig. 3a do not show such an obvious pattern associated with differential gene expression as seen for lineage-specific coding genes (Fig. 1). However, randomization tests revealed that the number of lineage-specific enhancers and promoters around lineage-specific lncRNAs are highly enriched compared with anywhere else in the genome (Figure S5a, b in Additional file 12). We then looked for overlap between disease-associated SNPs and lineage-specific lncRNAs and found many disease-associated SNPs (including immune-mediated diseases) that are enriched in the vicinity of Th1- and Th2-specific lncRNAs, suggesting they have a role in these diseases (Table 2).

\section{Functional characterization of identified IncRNAs}

Very little is known about the function of lncRNAs, but as shown in previous studies [47], co-expressed genes 


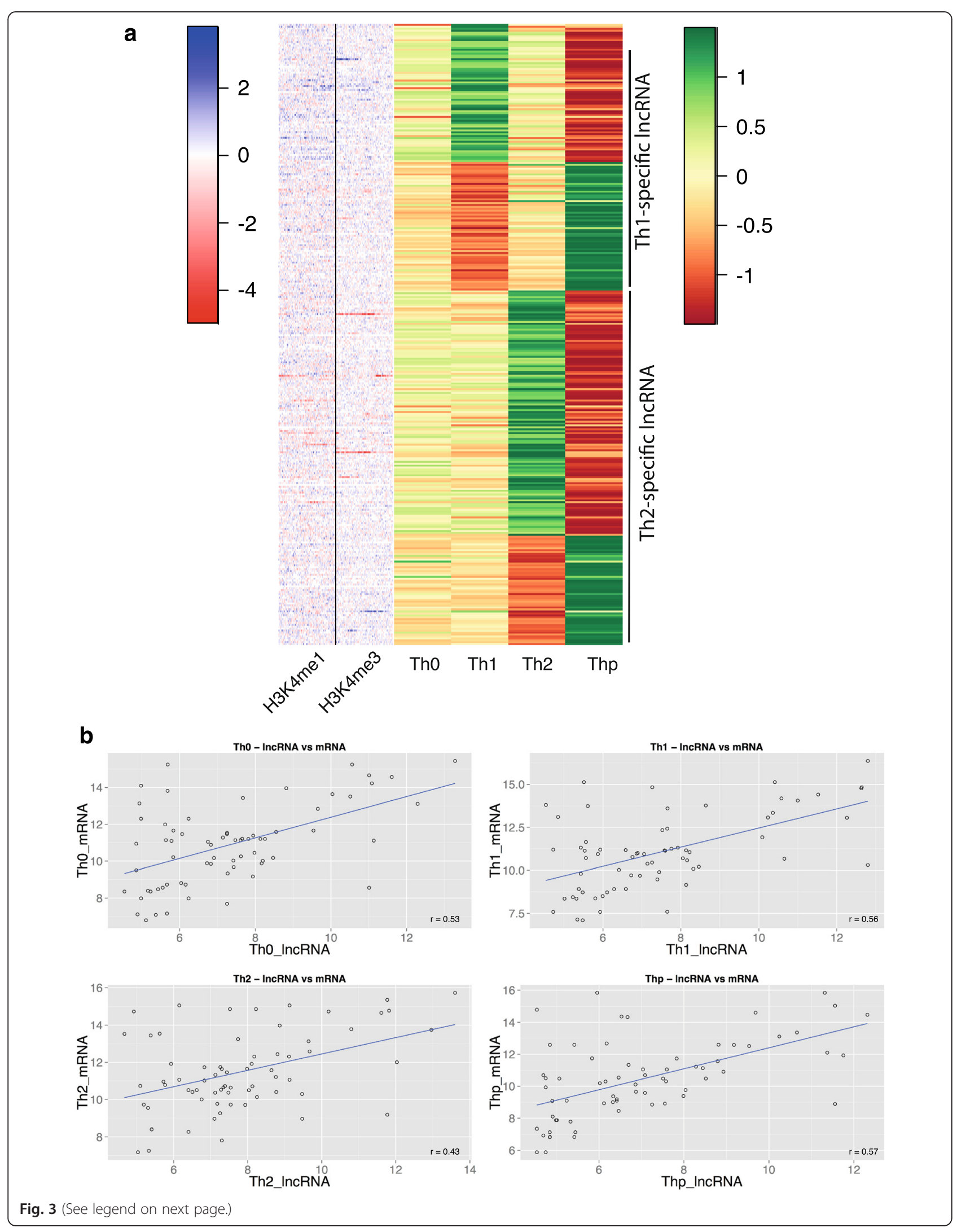


(See figure on previous page.)

Fig. 3 IncRNAs involved in early T-helper cell differentiation. a Heatmap showing expression and epigenetic profiles of Th1- and Th2-specific IncRNAs in T-helper cell subsets. Rows were first ordered based on log2 fold change and then by expression value. Normalized expression data from sequencing data were standardized using Z-score for visualization purposes. In the case of H3K4me1 and H3K4me3 marks, the ratio of tag counts at the transcription start site between Th1 and Th2 is shown. b Correlation plots of lineage-specific IncRNAs and lineage-specific genes in various T-helper cell subsets

participate in similar functions. Therefore, we constructed a co-expression network of lncRNAs and protein-coding genes. We then looked for GO terms enriched among the co-expressed genes and attributed the enriched GO terms to the lncRNAs. The GO terms enriched in lineage-

Table 2 Enrichment of disease-associated SNPs in Th1- and Th2-specific IncRNAs

\begin{tabular}{ll}
\hline Trait & $P$ value \\
\hline Th1-specific IncRNAs & \\
Biliary atresia & 0.007 \\
Hepatitis C & 0.008 \\
Breast neoplasms & 0.009 \\
Diabetes mellitus, type 1 & 0.015 \\
Cleft palate & 0.018 \\
Ovarian neoplasms & 0.023 \\
Diabetes mellitus, type 2 & 0.026 \\
Leukemia, lymphoid & 0.026 \\
Diabetic nephropathies & 0.029 \\
Hypothyroidism & 0.042 \\
Th2-specific IncRNAs & \\
Cleft lip & 0.001 \\
Lupus erythematosus, systemic & 0.002 \\
Parkinson disease & 0.003 \\
Stroke & 0.003 \\
Inflammatory bowel diseases & 0.0048 \\
Diabetes mellitus, type 2 & 0.048 \\
Biliary atresia & 0.032 \\
Osteoarthritis & 0.003 \\
Colitis, ulcerative & 0.005 \\
Breast neoplasms & 0.009 \\
Thyroid neoplasms & 0.009 \\
Esophagitis & 0.022 \\
Gallbladder diseases & 0.022 \\
Arthritis, rheumatoid & \\
\hline
\end{tabular}

specific lncRNAs are summarized in Additional file 13 and a complete list can be found in Additional file 14 . These GO terms aid in understanding the role of these lncRNAs in various biological processes.

\section{Discussion}

T-helper cell differentiation is a complex process and some previous studies have elucidated the genes involved in it [4-8]. Since most of the previous studies have used microarrays for global profiling of the transcriptome, they are limited by factors such as preselection bias and probe design [48]. In our study, we use multiple transcriptional profiling platforms to generate a high-confidence list of genes that are involved in T-helper cell speciation. In addition, we complement the high-confidence list of genes with a novel list of genes inferred from only next-generation sequencing data. This novel list has many genes that are not previously known in the context of T-helper cell differentiation.

In the process of obtaining these lineage-specific genes, we also compared the transcriptomic profiling platforms used. Our platform comparison results are in concordance with previously published studies $[49,50]$. The detection range of Illumina arrays is narrow compared with that of Affymetrix arrays and Illumina sequencing. These results aid in future experimental design, e.g., a next-generation sequencing platform is a good choice when one intends to study low-abundance genes.

To distinguish genuine expression from background noise, we generated present/absent calls for genes for each platform. In the case of Illumina arrays, welldefined negative probes enabled easy estimation of background and generation of detection $p$ values. In the case of Affymetrix arrays, the negative probes did not have a desirable behavior. Therefore, we have used Gaussian mixture modeling to estimate the probability of a gene being genuinely expressed. In the case of Illumina sequencing data, we used normalized data obtained after variance stabilization in estimating genuinely expressed genes using Gaussian mixture modeling.

Since next-generation sequencing data can be leveraged to quantify other transcripts, such as lncRNAs, we determined lineage-specific lncRNAs. Previous studies $[46,51]$ identified lncRNAs in completely differentiated T-helper cells but, to our knowledge, this is the first study with global profiles of lncRNAs involved in early stages of 
human Th1 and Th2 cell differentiation. Additionally, our analysis revealed the relationship between lineage-specific lncRNAs and lineage-specific gene expression and found that the lineage-specific lncRNAs and lineage-specific gene expression are positively correlated. The finding led us to speculate that some of the lncRNAs might be acting as either enhancer elements during T-helper cell differentiation as suggested by a previous study [9] or that the lncRNA and gene pair can be regulated by another factor as suggested by Hu et al. [51].

We also quantified the enrichment of disease SNPs in the vicinity of lineage-specific genes and lncRNAs. SNPs associated with both immune-mediated and nonimmune-mediated diseases were enriched around Th1and Th2-specific genes and lncRNAs. This suggests that besides immune-mediated ones, these elements might also be involved in other cellular processes. With recent advancements in genome-editing technologies like CRISPR/ Cas9, it will be possible to determine how a given SNP in a regulatory region might influence cellular functions involved in disease pathogenesis.

\section{Conclusion}

The results show the involvement of several new actors in the early differentiation of T-helper cells and the relationship between epigenetic factors and IncRNAs and their possible role in autoimmune diseases.

\section{Additional files}

\section{Additional file 1: Table S1. Reads obtained from next-generation} sequencing data. (XLSX $43 \mathrm{~kb}$ )

Additional file 2: Figure S1. Analysis design schematic. (PDF $133 \mathrm{~kb}$ )

Additional file 3: Figure S2. Comparison of transcriptional profiling platforms. a Genes determined to be present in T-helper cell subsets in the three platforms used for transcriptional profiling. b Gene expression density curves of T-helper cell subsets in the three platforms profiled. Only genes determined to be present were included. c Box plot of expression of genes in T-helper cell subsets based on their detection in the platforms used for transcriptional profiling. (PDF $117 \mathrm{~kb}$ )

Additional file 4: Figure S3. Distribution of lineage-specific genes in platforms when analyzed individually in each platform. (PDF 103 kb)

Additional file 5: Table S2. Table showing Jaccard similarity coefficients between platforms (XLSX $34 \mathrm{~kb}$ )

Additional file 6: Table S3. Table showing Pearson correlation coefficients between platforms. (XLSX $34 \mathrm{~kb}$ )

Additional file 7: Table S4. High confidence list of Th1- and Th2-specific genes in human. (XLSX $399 \mathrm{~kb}$ )

Additional file 8: Table S5. Novel list of Th1- and Th2-specific genes in human. (XLSX $122 \mathrm{~kb}$ )

Additional file 9: Table S6. List of Th1- and Th2-specific long non-coding RNAs. (XLSX 97 kb)

Additional file 10: Figure S4. Relative abundance of various IncRNA types along with protein-coding genes. The column names are as follows: AS anti sense IncRNA, AS_L lineage-specifc antisense IncRNA, linc long intergenic non-coding RNA, linc_L lineage-specific lincRNA, PT processed transcript, $P T \_L$ lineage-specific processed transcript, $S /$ sense intronic,
SI_L lineage-specific sense intronic, SO sense overlapping, SO_L lineagespecific sense overlapping, $P C$ protein-coding mRNA. (PDF $132 \mathrm{~kb}$ )

Additional file 11: Table S7. List of lineage-specific IncRNAs in the vicinity of lineage-specific protein coding genes. (XLSX $42 \mathrm{~kb}$ )

Additional file 12: Figure S5. Randomization test of epigenetic marks around lineage-specific IncRNAs. a Randomization test reveals that the number of lineage-specific enhancers around lineage-specific IncRNAs is more than anywhere else in the genome. b Randomization test reveals that the number of lineage-specific promoters around the lineage-specific IncRNAs is more than anywhere else in the genome. The distribution of enhancers or promoters in the vicinity of genes in the genome was determined by randomly picking the same number of genes as in the lineage-specific set. The red dashed line indicates the number of lineage-specific enhancers or promoters found in the vicinity of lineage-specific IncRNAs. (PDF $114 \mathrm{~kb}$ )

Additional file 13: Table S8. GO terms enriched in Th1- and Th2- specific IncRNAs. (XLSX 99 kb)

Additional file 14: Table S9. Predicted GO terms of lineage-specific IncRNAs. (XLSX $380 \mathrm{~kb}$ )

\section{Abbreviations}

EM: Expectation-Maximization; GO: Gene Ontology; IL: interleukin; IncRNA: long non-coding RNA; SNP: single nucleotide polymorphism; Th: T-helper.

\section{Competing interests}

The authors declare that they have no competing interests.

\section{Authors' contributions}

Study concept and design: KK, HL, and RL. Data generation: KK, ST, RL, DH, $\mathrm{BR}, \mathrm{HL}$, and RL. Data analysis: KK, HM, UU, HM, AL, HL, and RL. Manuscript drafting: KK, ST, HL, and RL. All authors read and approved the final manuscript.

\section{Acknowledgements}

The authors would like to thank Marjo Hakkarainen, Sarita Heinonen, Päivi Junni and Elina Pietilä and the personnel of The Finnish Microarray and Sequencing Center (FMSC) at Turku Center for Biotechnology for excellent technical assistance, FMSC belongs to Biocenter Finland, whose support is acknowledged. We acknowledge the computational resources provided by the Aalto Science-IT project. This research was supported by research grants from the Turku Doctoral Programme in Molecular Medicine (TuDMM) to KK, European Commission Seventh Framework grant EC-FP7-SYBILLA-201106 to $\mathrm{RL}$ and $\mathrm{HL}$, the Academy of Finland (Centre of Excellence in Molecular Systems Immunology and Physiology Research, 2012-2017, grant 250114) to RL and HL and the Sigrid Jusélius Foundation.

\section{Author details}

${ }^{1}$ Turku Centre for Biotechnology, University of Turku and Åbo Akademi University, Turku, Finland. ²Department of Computer Science, Aalto University School of Science, Espoo, Finland. ${ }^{3}$ Division of Medical Genetics, Department of Medicine, University of Washington School of Medicine, Seattle, WA 98195, USA. ${ }^{4}$ Department of Genome Sciences, University of Washington School of Medicine, Seattle, WA 98195, USA. ${ }^{5}$ Ludwig Institute for Cancer Research, La Jolla, CA 92093, USA. ${ }^{6}$ Department of Cellular and Molecular Medicine, Institute of Genomic Medicine and Moores Cancer Center, University of California, San Diego, La Jolla, CA 92093, USA.

Received: 1 August 2015 Accepted: 2 November 2015 Published online: 20 November 2015

\section{References}

1. Zhernakova A, van Diemen CC, Wijmenga C. Detecting shared pathogenesis from the shared genetics of immune-related diseases. Nat Rev Genet. 2009; 10:43-55. 
2. Cho S-H, Stanciu LA, Holgate ST, Johnston SL. Increased interleukin-4, interleukin-5, and interferon-gamma in airway CD4+ and CD8+ T cells in atopic asthma. Am J Respir Crit Care Med. 2005;171:224-30.

3. Woodfolk JA. T-cell responses to allergens. J Allergy Clin Immunol. 2007;119:280-94.

4. Lund $\mathrm{R}$, Ahlfors $\mathrm{H}$, Kainonen $\mathrm{E}$, Lahesmaa A-M, Dixon C, Lahesmaa R. Identification of genes involved in the initiation of human Th1 or Th2 cell commitment. Eur J Immunol. 2005:35:3307-19.

5. Lund RJ, Löytömäki M, Naumanen T, Dixon C, Chen Z, Ahlfors H, et al. Genome-wide identification of novel genes involved in early Th1 and Th2 cell differentiation. J Immunol. 2007;178:3648-60.

6. Chtanova T, Newton R, Liu SM, Weininger L, Young TR, Silva DG, et al. Identification of T cell-restricted genes, and signatures for different $T$ cell responses, using a comprehensive collection of microarray datasets. J Immunol. 2005:175:7837-47.

7. Rogge L, Bianchi E, Biffi M, Bono E, Chang S-YP, Alexander $\mathrm{H}$, et al. Transcript imaging of the development of human T helper cells using oligonucleotide arrays. Nat Genet. 2000;25:96-101.

8. Lu B, Zagouras P, Fischer JE, Lu J, Li B, Flavell RA. Kinetic analysis of genomewide gene expression reveals molecule circuitries that control T cell activation and Th1/2 differentiation. Proc Natl Acad Sci U S A. 2004;101: 3023-8

9. Ørom UA, Derrien T, Beringer M, Gumireddy K, Gardini A, Bussotti G, et al. Long noncoding RNAs with enhancer-like function in human cells. Cell. 2010;143:46-58.

10. Mudge JM, Frankish A, Harrow J. Functional transcriptomics in the post-ENCODE era. Genome Res. 2013;23:1961-73.

11. Murphy KM, Reiner SL. The lineage decisions of helper T cells. Nat Rev Immunol. 2002;2:933-44.

12. Wilson CB, Rowell E, Sekimata M. Epigenetic control of T-helper-cell differentiation. Nat Rev Immunol. 2009;9:91-105.

13. Kanno Y, Vahedi G, Hirahara K, Singleton K, O'Shea JJ. Transcriptional and epigenetic control of $T$ helper cell specification: molecular mechanisms underlying commitment and plasticity. Annu Rev Immunol. 2012;30:707-31

14. Aune TM, Collins PL, Chang S. Epigenetics and T helper 1 differentiation. Immunology. 2009;126:299-305.

15. Hawkins RD, Larjo A, Tripathi SK, Wagner U, Luu Y, Lönnberg T, et al. Global chromatin state analysis reveals lineage-specific enhancers during the initiation of human T helper 1 and T helper 2 cell polarization. Immunity. 2013:38:1271-84

16. Rinn JL, Chang HY. Genome regulation by long noncoding RNAs. Annu Rev Biochem. 2012:81:145-66.

17. Irizarry RA, Hobbs B, Collin F, Beazer-Barclay YD, Antonellis KJ, Scherf U, et al. Exploration, normalization, and summaries of high density oligonucleotide array probe level data. Biostatistics. 2003;4:249-64.

18. Pagani M, Rossetti G, Panzeri I, de Candia P, Bonnal RJP, Rossi RL, et al. Role of microRNAs and long-non-coding RNAs in CD4(+) T-cell differentiation Immunol Rev. 2013:253:82-96.

19. Gentleman R, Carey V, Huber W, Hahne F. Package "genefilter". 2012. https://www.bioconductor.org/packages/release/bioc/html/genefilter.html.

20. Benaglia T, Chauveau D, Hunter D, Young D. mixtools: an R package for analyzing finite mixture models. J Stat Softw. 2009:32:1-29.

21. Lee HJ, Suk JE, Patrick C, Bae EJ, Cho JH, Rho S, et al. Direct transfer of -synuclein from neuron to astroglia causes inflammatory responses in synucleinopathies. J Biol Chem. 2010;285:9262-72.

22. Smyth GK. Linear models and empirical bayes methods for assessing differential expression in microarray experiments. Stat Appl Genet Mol Biol. 2004;3:Article 3.

23. Du P, Kibbe WA, Lin SM. lumi: a pipeline for processing Illumina microarray. Bioinformatics. 2008;24:1547-8.

24. Patel RK, Jain M. NGS QC Toolkit: a toolkit for quality control of next generation sequencing data. PLoS One. 2012;7:e30619.

25. Kim D, Pertea G, Trapnell C, Pimentel H, Kelley R, Salzberg SL. TopHat2: accurate alignment of transcriptomes in the presence of insertions, deletions and gene fusions. Genome Biol. 2013:14:R36.

26. Anders $\mathrm{S}, \mathrm{Huber}$ W. Differential expression analysis for sequence count data. Genome Biol. 2010;11:R106.

27. Harrow J, Frankish A, Gonzalez JM, Tapanari E, Diekhans M, Kokocinski F, et al. GENCODE: the reference human genome annotation for The ENCODE Project. Genome Res. 2012;22:1760-74
28. Alexa A, Rahnenführer J, Lengauer T. Improved scoring of functional groups from gene expression data by decorrelating GO graph structure. Bioinformatics. 2006;22:1600-7.

29. Pink M, Ratsch BA, Mardahl M, Schröter MF, Engelbert D, Triebus J et al. Identification of two regulatory elements controlling Fucosyltransferase 7 transcription in murine CD4+ T cells. Mol Immunol. 2014;62:1-9.

30. Chen G-Y, Osada H, Santamaria-Babi LF, Kannagi R. Interaction of GATA-3/T-bet transcription factors regulates expression of sialyl Lewis $X$ homing receptors on Th1/Th2 lymphocytes. Proc Natl Acad Sci U S A. 2006;103:16894-9

31. Schrum S, Probst P, Fleischer B, Zipfel PF. Synthesis of the CC-chemokines MIP-1alpha, MIP-1beta, and RANTES is associated with a type 1 immune response. J Immunol. 1996;157:3598-604.

32. Vetter ML, Johnson ME, Antons AK, Unutmaz D, D'Aquila RT. Differences in APOBEC3G expression in CD4+ T helper lymphocyte subtypes modulate HIV-1 infectivity. PLoS Pathog. 2009;5:e1000292.

33. Mohanram V, Sköld AE, Bächle SM, Pathak SK, Spetz A-L. IFN-a induces APOBEC3G, F, and A in immature dendritic cells and limits HIV-1 spread to CD4+ T cells. J Immunol. 2013;190:3346-53.

34. Bouwmeester T, Bauch A, Ruffner H, Angrand P-O, Bergamini G, Croughton $\mathrm{K}$, et al. A physical and functional map of the human TNF-alpha/NF-kappa B signal transduction pathway. Nat Cell Biol. 2004;6:97-105.

35. Tuomela S, Salo V, Tripathi SK, Chen Z, Laurila K, Gupta B, et al. Identification of early gene expression changes during human Th17 cell differentiation. Blood. 2012;119:e151-60

36. Elo LL, Järvenpää H, Tuomela S, Raghav S, Ahlfors H, Laurila K, et al. Genome-wide Profiling of interleukin-4 and STAT6 transcription factor regulation of human Th2 cell programming. Immunity. 2010;32:852-62.

37. Belperio JA, Dy M, Murray L, Burdick MD, Xue YY, Strieter RM, et al. The role of the Th2 CC chemokine ligand CCL17 in pulmonary fibrosis. J Immunol. 2004:173:4692-8.

38. Staples K, Hinks TSC, Ward JA, Gunn V, Smith C, Djukanović R. Phenotypic characterization of lung macrophages in asthmatic patients: overexpression of CCL17. J Allergy Clin Immunol. 2012;130:1404-12. e7.

39. Nakazato J, Kishida M, Kuroiwa R, Fujiwara J, Shimoda M, Shinomiya N Serum levels of Th2 chemokines, CCL17, CCL22, and CCL27, were the important markers of severity in infantile atopic dermatitis. Pediatr Allergy Immunol. 2008;19:605-13.

40. Wirnsberger G, Hebenstreit D, Posselt G, Horejs-Hoeck J, Duschl A. IL-4 induces expression of TARC/CCL17 via two STAT6 binding sites. Eur J Immunol. 2006:36:1882-91.

41. Cerasoli DM, Kelsoe G, Sarzotti M. CD4+ Thy1- thymocytes with a Th-type 2 cytokine response. Int Immunol. 2001;13:75-83.

42. Watanabe T, Kitani A, Murray PJ, Strober W. NOD2 is a negative regulator of Toll-like receptor 2-mediated T helper type 1 responses. Nat Immunol. 2004;5:800-8.

43. Magalhaes JG, Fritz JH, Le Bourhis L, Sellge G, Travassos LH, Selvanantham T, et al. Nod2-dependent Th2 polarization of antigen-specific immunity. J Immunol. 2008;181:7925-35

44. Heintzman ND, Stuart RK, Hon G, Fu Y, Ching CW, Hawkins RD, et al. Distinct and predictive chromatin signatures of transcriptional promoters and enhancers in the human genome. Nat Genet. 2007:39:311-8,

45. Derrien $T$, Johnson $R$, Bussotti $G$, Tanzer A, Djebali S, Tilgner $H$, et al The GENCODE v7 catalog of human long noncoding RNAs: analysis of their gene structure, evolution, and expression. Genome Res. 2012;22:1775-89.

46. Ranzani V, Rossetti G, Panzeri I, Arrigoni A, Bonnal RJP, Curti S, et al. The long intergenic noncoding RNA landscape of human lymphocytes highlights the regulation of T cell differentiation by linc-MAF-4. Nat Immunol. 2015:16:318-25.

47. Lee HK, Hsu AK, Sajdak J, Qin J, Pavlidis P. Coexpression analysis of human genes across many microarray data sets. Genome Res. 2004;14:1085-94.

48. $t$ Hoen PAC, Ariyurek Y, Thygesen HH, Vreugdenhil E, Vossen RHAM, de Menezes RX, et al. Deep sequencing-based expression analysis shows major advances in robustness, resolution and inter-lab portability over five microarray platforms. Nucleic Acids Res. 2008;36:e141-1.

49. Konopka G, Friedrich T, Davis-Turak J, Winden K, Oldham MC, Gao F, et al. Human-specific transcriptional networks in the brain. Neuron. 2012;75:601-17. 
50. Beyer M, Mallmann MR, Xue J, Staratschek-Jox A, Vorholt D, Krebs W, et al. High-resolution transcriptome of human macrophages. PLoS One. 2012;7:e45466.

51. Hu G, Tang Q, Sharma S, Yu F, Escobar TM, Muljo SA, et al. Expression and regulation of intergenic long noncoding RNAs during T cell development and differentiation. Nat Immunol. 2013;14:1190-8.

Submit your next manuscript to BioMed Central and take full advantage of:

- Convenient online submission

- Thorough peer review

- No space constraints or color figure charges

- Immediate publication on acceptance

- Inclusion in PubMed, CAS, Scopus and Google Scholar

- Research which is freely available for redistribution 